\title{
Hobby preferences and physical activity participation among children with and without autism spectrum disorder
}

\author{
Scott Russell ${ }^{1}$, Sean Healy ${ }^{2 *}$, and Rock E. Braithwaite ${ }^{3}$ \\ Received: 24-August-2018; Accepted: 25 $5^{\text {th }}$ October 2018; Published: $16^{\text {th }}$ January 2019
}

\begin{abstract}
Children diagnosed with Autism Spectrum Disorder (ASD) exhibit low physical activity levels and are at an increased risk for health problems related to sedentary behaviors. Hobby preferences have been shown to influence physical activity participation. The primary purpose of this study was to examine the hobby preferences of children with ASD and determine if physical activity levels differed, depending on the nature (active versus sedentary) of their hobby preferences. A secondary purpose was to describe the hobby preferences of children with ASD. Participants included 86 nine-year-old children with and without ASD. Physical activity levels were reported by the parents using the Godin Leisure Time Exercise Questionnaire and children reported on their hobby preferences. Chi-square, T-test and ANOVA analysis indicated children with ASD were less active than their typically developing (TD) peers, less likely to prefer active hobbies, and lastly, physical activity of children with ASD with active hobbies was not significantly less than children with ASD with sedentary hobbies. This study also provides frequency data on the hobby preferences of children with ASD.
\end{abstract}

Keywords: Autistic; exercise; active; health; sedentary behavior; video games; screen-time

\section{Introduction}

Autism Spectrum Disorder (ASD) is a complex neurological and developmental disorder, diagnosed by the presence of symptoms from two categories (American Psychiatric Association, 2013): (A) a persistent deficit in social communication and social interaction across multiple contexts. These deficits include having difficulty with social reciprocity, non-verbal communicative behaviors, and the ability to develop, maintain, and understand relationships, and (B) the presence of at least two symptoms related to restrictive or repetitive patterns of behavior, activity, or interests. Possible symptoms or behaviors include repetitive motor movements or speech, an insistence on sameness or ritualized patterns of verbal or nonverbal behavior, fixated interests of abnormal intensity, and hyper- or hyperactivity to sensory input. ASD has received an increased focus in research over the past 20 years due to the alarming increase in diagnosis rates. In 2000, it was estimated that roughly 1 in 150 children were diagnosed while today 1 in 68 children are thought to be diagnosed with the disorder (Christensen, 2016). ASD is prevalent in all ethnic groups and is four times as likely in boys as in girls (Christensen, 2016). Cognitive skills can vary for individuals diagnosed with ASD and some have an increased risk of medical conditions such as seizures and sleep problems as well as other genetic or developmental disorders (Cohen et al., 2005). Decreased motor control can also affect individuals with ASD (Must, Phillips, Curtin, \& Bandini, 2015).

Research on physical activity levels of youth with ASD is somewhat conflicting. Research has shown participation levels of children with ASD to be significantly less when compared to TD peers (Pan, Tsai, Chu, \& Hsieh, 2011; Tyler, Macdonald, \& Menear, 2014; Corvey, Menear, Preskitt, Goldfarb, \& Menachemi, 2016) and the physical activity guidelines (Memari et al., 2015). Conversely, some research suggests activity levels are comparable (Corvey et al., 2016), including when controlling for intelligence quotient, ASD severity, and gender (Macdonald, Esposito, \& Ulrich, 2011). Research on sedentary behavior is more conclusive, albeit more scarce, demonstrating youth with 
ASD to participate in significantly more screen-time than youth with ASD (Must et al, 2014; Healy, Haegele, Grenier, \& Garcia, 2017). For example, Must et al., (2014) revealed that more children with ASD (aged 3 to 11) exceeded the recommended two hours of screen-time on weekdays and at weekends in comparison to TD children (weekdays: $63 \%$ vs $40 \%$, and weekend: $90 \%$ vs $62 \%$ ). While a consensus has not yet been reached on the physical activity levels of children with ASD, it is clear that their individual traits and symptoms related to their diagnosis may have a significant impact on their choice to be active.

Research has revealed barriers to physical activity among youth with ASD to exist at all levels of the ecological model. Child and family level barriers are reported by parents and children including behavioral problems, motor skills deficits, feeling tired, sensory-processing issues, a lack of time, and fear of injury (Stanish et al., 2015; Must et al., 2015; Healy, Msetfi, \& Gallagher, 2013). In addition, children with ASD often report a preference for sedentary behaviors, particularly screenbased activities (Stanish et al, 2015; Healy et al., 2017, Obrusnikova \& Cavalier, 2011), and parental time constraints (Obrusnikova, 2012). Social barriers including a lack of experts (Must et al., 2015) and a lack of peer exercise partners (Obrusnikova, et al., 2012) were noted as social barriers. Finally, environmental barriers identified as impeding physical activity participation among this population include inclement weather (Stanish, et al, 2015; Obrusnikova \& Cavalier, 2011), a lack of community programs, transportation, safe equipment, and high cost (Must et al., 2015).

Due to the belief that hobby preferences affect activity engagement, there has been a multitude of studies examining the hobby preferences of youth. One such study of the preferences of middle school students found that individuals may become disengaged in activity units they do not prefer (Hill \& Hannon, 2008). Middle school students, in the US, were more likely to prefer familiar or mainstream activities such as basketball or football as opposed to less familiar activities such as team handball or orienteering. Notable differences between genders were also found among middle school children with a majority of girls selecting activities such as yoga or gymnastics that were not within the majority of the boys' preference. Lastly, Hill \& Hannon (2008) found that overall interest in all activities declined as they approached higher grade levels, regardless of gender. The research based on activity preferences among youth often focuses on differences between genders; for example, Hill and Cleven (2005) compared the preferences of 9th grade males and females, and found significant differences between physical hobby preferences in boys and girls. Girls showed more interest in noncontact activities and a large portion of girls were described as having no opinion towards physical activity or physical education (Hill \& Cleven, 2005). This research is useful for intervention development and program planning for the TD youth population, but the findings may not be generalizable toward other specific sub-groups of youth - for example, youth with ASD.

Due to the unique characteristics of individuals with ASD - including social, behavioral, and sensory differences - hobby preferences among children with ASD may differ from children without ASD, as their experiences of the setting may differ. In addition, due to the tendency for children with ASD to be more sedentary, an understanding of their hobby preferences is warranted. An understanding of their hobby preferences will allow for more tailored, relevant interventions and program curriculums to be developed. Incorporating their interests may lead to an increase in physical activity engagement and achievement of the associated health benefits. However, research on activity preferences among youth with ASD is scarce. To the best knowledge of the authors, only Stanish et al., (2015) sought the perspective of adolescents with ASD (aged 13 to 21) regarding their preference of physical activities (for example where, and with whom, they most like to participate) and their enjoyment of non-physical activities. Differences in perceptions of activities did emerge. For example, $25 \%$ of adolescences with ASD reported to prefer playing sport or exercise, in comparison to $58 \%$ of the TD adolescents. Thirty-five percent of adolescents with ASD reported a dislike of team sports, in comparison to $5 \%$ of TD participants. Regarding enjoyment of non-physical activities (watching television and playing video games, reading, and arts and crafts), no significant differences were evident between groups. Further research is required to (1) examine activity preferences of younger children with ASD; (2) examine how physical activity levels may change depending on activity preferences, and (3) delineate the activity preferences among youth with ASD. 
The primary purpose of this study was to examine the hobby preferences of nine-year old children with ASD and determine if physical activity levels differed, depending on the nature of their hobby preferences. A secondary purpose was to describe the hobby preferences of children with ASD.

\section{Materials and Methods}

\section{Participants and data collection}

The first wave of the nationally representative data set, Growing Up in Ireland (GUI) provided data on physical, behavioral, psychological, environmental, and cognitive outcomes for 9-year olds living in Ireland. Using a systematic stratified sampling procedure, 8570 participants $(n=8570)$ from 910 schools across Ireland were recruited. The data set was collected between September 2007 and June 2008. Ethical approval was given by the Research Ethics Committee of the Irish Health Research Board. Both parents and children completed questionnaires on a number of outcomes regarding the child's characteristics, experiences, and development. Teachers and school principals were also tasked with providing information on the child's social, emotional, cognitive development, and school characteristics. Response rates at the school level were $82 \% ; 57 \%$ at the family level. The technical report (Murray et al., 2011) describes various efforts made to elicit an accurate response from participants. For example, the interviewer received training emphasizing the importance of establishing a good rapport, the power imbalance that may exist between interviewer and participant, and strategies to put the child at ease (for example asking them about their favorite activities) (Murray et al., 2011). In addition, the interviewer was instructed to explain a question when not understood by the respondent, and prompt cards were available with the possible answers if necessary (Murray et al., 2011). More information regarding the data set, including methodology and framework, can be found in Murray et al. (2011).

The current study included 86 participants: 43 of the participants were children diagnosed with ASD while 43 typically developing (TD) children were randomly selected for comparison purposes. Each group consisted of 37 males and 6 females. Children were placed in the 'ASD group' only if their primary caregiver reported an ASD diagnosis was made by a medical professional.

\section{Measures}

Multiple parent and self-reported measures were included in the analysis. The Leisure Time Exercise Questionnaire (Godin \& Shepard, 1985) was utilized to measure parental-report physical activity of the children. Participation in moderate to vigorous physical activity (MVPA) was assessed by posing the following question to the parent. "How many times in the past 14 days, has the Study Child done at least 20 minutes of hard exercise to make them breathe fast and make their heart beat faster? Activity examples were provided such as playing soccer, jogging, or cycling at an accelerated pace. Participants responded using a five-point scale, ranging from 1 (none) to 5 (9 or more days). This measure has demonstrated concurrent validity when used in previous research that measured maximum oxygen intake (VO2 max) and muscular endurance (Cadogen, Keane \& Kearney 2014; Godin, Jobin, Bouillon, 1986) with acceptable test-retest reliability (Sallis et al., 1993). A self-report physical activity measure was also utilized, posed as "over the past 7 days on how many days were you physically active for a total of at least 60 minutes per day?" Responses were recorded numerically.

To assess hobby preferences among the participants, the question "what is your favorite hobby or activity?" was posed to the child, and they were able to choose from 32 hobby types. Two researchers separately categorized the 32 hobbies into three distinct groups: active, sedentary, and unknown (not clearly active or sedentary). A consensus was reached through discussion when any differences of opinion were found. Examples of active hobbies included soccer, swimming, cycling, with fourteen active activities identified in total. Sedentary activities included hobbies and activities such as arts \& crafts, playing an instrument, and watching TV, with twelve sedentary hobbies identified in total. Six activities were determined by both researchers to be too ambiguous to be categorized as active or sedentary with confidence and thus were labeled unknown. Frequency data 
on the hobby preferences of the sample group was compiled using results from the previously stated "favorite hobby" question and can be found in Table 1.

Table 1. Hobby preferences among children with and without ASD

\begin{tabular}{ccc}
\hline & ASD & TD \\
& $(\mathrm{n}=43)$ & $(\mathrm{n}=43)$ \\
& $\mathrm{n}(\%)$ & $1 \%)$ \\
\hline Playing video games & $10(23.3)$ & $11(25.6)$ \\
Football or soccer & $5(11.6)$ & $4(9.3)$ \\
Reading or writing & $3(7)$ & 0 \\
Arts, crafts, and cookery & $3(7)$ & $2(4.7)$ \\
Interacting with friends other than sport & $3(7)$ & $4(9.3)$ \\
Sport or physical exercise not otherwise specified & $3(7)$ & $1(2.3)$ \\
Watching TV or DVDs & $2(4.7)$ & 0 \\
Non-physical activities such as go-karting & $2(4.7)$ & $4(9.3)$ \\
Swimming & $2(4.1)$ & $1(2.3)$ \\
Horse or pony riding & $1(2.3)$ & $3(7)$ \\
Other individual sport & $1(2.3)$ & 0 \\
Dancing & $1(2.2)$ & $2(4.7)$ \\
Other hobby, e.g. Collecting & $1(2.3)$ & $1(2.3)$ \\
Basketball & $1(2.3)$ & $1(2.3)$ \\
Gymnastics & $1(2.3)$ & $3(7)$ \\
Hurling or Camogie & 0 & $3(7)$ \\
Rugby & 0 & $1(2.3)$ \\
Other team sport & 0 & $1(2.3)$ \\
Cycling & 0 &
\end{tabular}

\section{Statistical Analysis}

Descriptive statistics were provided for the nature of the hobby preferences (active versus sedentary) and physical activity levels for both groups, with ASD and the TD group. Chi square analysis was used to compare the nature of hobby preferences between children with and without ASD and an independent samples T-test was used to compare physical activity levels between groups. Among the children with ASD, an independent samples T-test was used to compare physical activity levels between children with active hobby preferences and sedentary hobby preferences. To compare PA levels between children with and without ASD, with active hobby preferences or sedentary hobby preferences, an ANOVA was utilized. In the event that there is a violation of the assumption of normality of variance, a non-parametric Kruskal-Wallis test will be used. When the equality of variance assumption was violated, a nonparametric equivalent analysis was used. Finally, descriptive statistics were provided for hobby preferences for children with ASD.

\section{Results}

\section{Demographics}

Groups were not significantly different based on equivalized-household income (weighted to account for differences in size and composition of households in terms of the number of adults and children in the home). Mean income among the families of the children with ASD was $€ 21,448$ (SD = 11705.47 ) versus $€ 23,171.38$ ( $\mathrm{SD}=13665.12$ ) among the TD group; $\mathrm{F}(1,84)=.644, \mathrm{p}=-.425$.

\section{Physical Activity Levels}

Parent and self-reported measures of physical activity were examined to evaluate differences between groups. Parents reported on the number of times their child participated in MVPA over the 
past 14 days. It was revealed that children with ASD engaged in fewer days of MVPA (M = 3.77, SD $=1.34)$ than their typically developing peers $(\mathrm{M}=4.40, \mathrm{SD}=1.00), \mathrm{t}(84)=-2.46, \mathrm{p}=.016)$. Childreported data indicated that children with ASD engaged in fewer days of 60 minutes of physical activity during the week $(\mathrm{M}=4.03, \mathrm{SD}=.2 .323)$ than their typically developing peers $(\mathrm{M}=5.58, \mathrm{SD}=$ $2.206), t(80)=-3.11, p=.003$. There was a significant moderate positive correlation between selfreported number of days with 60 minutes of physical activity per week, and parent-report number of days of MVPA in the previous two weeks $(\mathrm{r}=.284, \mathrm{p}=.010)$.

\section{Hobby Preferences and Physical Activity Levels}

A Chi-square test revealed children with ASD and TD children significantly differed in respect to having active versus sedentary hobby preferences $(\mathrm{X} 2(2, \mathrm{~N}=82)=12.35, \mathrm{p}<.001)$. Among the children with ASD, $61.5 \%(n=24)$ reported a favorite hobby that was sedentary in nature while $38.5 \%$ $(n=15)$ preferred an active hobby. Four children with ASD failed to respond to this question or selected 'other' for their answer. In the group of TD children $76.7 \%(n=33)$ reported to have an active hobby, while $23.3 \%(n=10)$ reported a sedentary hobby.

To compare physical activity levels between children with and without ASD, who had active or sedentary hobby preferences, an ANOVA was conducted using the four groups. The four groups were, (1) children with ASD who had an active hobby preference $(n=15),(2)$ children with ASD with a sedentary hobby preference $(n=24),(3)$ TD children with an active hobby preference $(n=33)$, and $(4)$ TD children with a sedentary hobby preference $(n=10)$. Due to a violation of the assumption of normality of variance, a non-parametric Kruskal-Wallis test was conducted to assess differences in parent-reported vigorous physical activity between groups; no significant differences were revealed, $p=.083$. See Table 2 for physical activity levels between groups. Second, physical activity levels were compared based on child-report data (days of physical activity over the past week). Significant differences between the four groups were revealed $(\mathrm{F}=3.62, \mathrm{p}=.017)$. A post-hoc Bonferroni correction showed that TD children who had an active hobby preference were significantly more active $(5.76, \pm 2.151)$, than children with ASD who had a sedentary hobby preference $(3.96, \pm 2.477)$, $\mathrm{p}=.019$.

Table 2. Physical Activity levels among groups by hobby preference

\begin{tabular}{cccccc}
\hline & $\begin{array}{c}\text { ASD with } \\
\text { Active Hobby } \\
\text { Preference }\end{array}$ & $\begin{array}{c}\text { ASD with } \\
\text { Sedentary } \\
\text { Hobby } \\
\text { Preference } \\
(\mathrm{n}=24)\end{array}$ & $\begin{array}{c}\text { TD with } \\
\text { active hobby } \\
\text { preference } \\
\mathrm{m}(\mathrm{SD})\end{array}$ & $\begin{array}{c}\text { TD with } \\
\text { sedentary } \\
\text { hobby } \\
\text { preference } \\
(\mathrm{n}=11)\end{array}$ & p-value \\
$\begin{array}{c}\text { Vigorous } \\
\text { physical }\end{array}$ & $4.07( \pm 1.16)$ & $3.67( \pm 1.40)$ & $4.48( \pm 0.80)$ & $4.10( \pm 1.52)$ & .083 \\
$\begin{array}{c}\text { activity over } \\
\text { 14 days }\end{array}$ & & & & & \\
$\begin{array}{c}\text { Number of } \\
\text { days active in } \\
\text { one week }\end{array}$ & $4.00( \pm 2.17)$ & $3.96( \pm 2.48)$ & $5.69( \pm 4.79)$ & $5.00( \pm 2.40)$ & .017 \\
\hline
\end{tabular}

\section{Hobby Preferences}

Frequency data were collected on the hobby preferences of children with and without ASD. Among the children with ASD, the most frequently reported preferred hobby was playing video games, reported by $23.3 \%$ of the sample $(n=10)$. Conversely, playing video games was only cited as being a preferring hobby by $2.3 \%(n=1)$ of the TD sample. The next hobby type chosen most frequently by the group with ASD was football or soccer, reported by $11.6 \%(n=5)$; in comparison, among the TD group, this hobby was reported as being the preferred hobby of choice by $25.6 \%(n=11)$. 
It's interesting to note, that among the TD group, the three most frequently reported hobby preferences were all active; soccer or football, sport or activities not otherwise specified, and swimming. See Table 1 for an overview of hobby preferences.

\section{Discussion}

The primary purpose of this study was to examine the hobby preferences of children with ASD and determine if physical activity levels differed, depending on the nature of their hobby preferences. The results revealed that children diagnosed with ASD reported more sedentary hobby preferences than their TD peers. Over $60 \%$ of children with ASD reported a preference for a sedentary hobby, while more than $75 \%$ of TD children preferred an active hobby. This result is reflective of the finding by Stanish et al., (2015), in which $58 \%$ of TD adolescents reported a preference of 'sport or exercise' in comparison to only $25 \%$ of adolescents with ASD; the majority of adolescents with ASD $(75 \%)$ preferred to do 'something else' in their free time. Various reasons may exist for this preference for more sedentary pursuits; first, due to the unique social challenges experienced by children with ASD, traditional sports that depend heavily on teamwork and communication may be less appealing or perceived to be too difficult; indeed, $77 \%$ of parents of children with ASD reported difficulties with social skills as being a barrier to physical activity participation. Reports from children with ASD themselves, echo this sentiment; for example, children with ASD by Healy, Msetfi, \& Gallagher (2013) spoke of exclusion and experiencing bullying in physical activity environments. Furthermore, regarding social interaction, previous research has shown that fewer children with ASD believed physical activity to be an opportunity to make friends than their TD peers (Stanish et al., 2015). Motor skills deficits - considered pervasive among children with ASD (Fournier, Hass, Naik, Lodha, \& Cauraugh, 2010) - may also contribute to an aversion to physical activity as a hobby of choice. Fortytwo percent of parents in research by Must et al. (2015) report motor deficits as a barrier to physical activity participation. Feeling tired, sensory-processing issues, and fear of injury, all noted as barriers to physical activity (Stanish et al., 2015; Must et al., 2015; Healy, Msetfi, \& Gallagher, 2013) may also contribute to the desire for more sedentary activities; activities that are typically less dynamic and social, and where the sensory stimulation is more predictable and controlled. Another consideration is the preference for routines or ritualistic behaviors that individuals with ASD often exhibit. This may hinder their ability, and willingness, to participate in unfamiliar activities or develop new motor skills.

Interestingly, the activity levels between children with ASD who preferred active hobbies and those with ASD who preferred sedentary hobbies were not significantly different, based on parent reported data. Children with ASD who preferred sedentary hobbies exhibited similar physical activity levels as children with ASD who preferred active hobbies. Some possible reasons for this may include the parents' influence on activity at young ages by serving as "gatekeepers" in which they determine what activities and resources are available to the child (Welk, Wood, \& Morss, 2003). This influence may mitigate the impact of child's personal hobby preferences on their actual behaviors. With parents playing a key role in influencing activity levels of young children, it is important to continue to support parents' efforts to promote physical activity. However, it is also important to be cognizant that as a child with ASD ages and gains autonomy, their own preferences may be more influential on their health behaviors, including physical activity. Indeed, research has demonstrated physical activity levels of younger children with and without ASD to be comparable (e.g. Bandini et al., 2013), yet physical activity decreases significantly as they enter adolescence, and the disparity in activity levels compared to TD adolescents appears to grow (Healy, Haegele, Grenier, \& Garcia, 2017; McDonald, Esposito, \& Ulrich, 2011; Pan \& Frey, 2006). Future research should examine the influence of the parents over health behaviors of youth with ASD, and how this influence may change as the child ages and gains more autonomy.

A secondary purpose of this study was to provide frequency data on the hobby preferences of children with ASD. In line with the aim of previous research (e.g. Stanish et al., 2017), this was determined to be a worthy purpose, as an understanding of hobby preferences of children with ASD may inform intervention development. As previously stated, playing video games was the most 
popular hobby among children with ASD; identified as the preferred hobby by $23.3 \%$ of children with ASD, in comparison to just $2.3 \%$ of the TD sample. Indeed, previous research on sedentary behavior has reflecting this phenomenon; in research by Must et al., (2014) children with ASD participated in an hour more of sedentary behaviors on weekdays, and most of this difference was due to screen-time. Also, children with ASD in research by Stanish et al., (2017) most commonly reported participate in video games. Some hypotheses are posited as to why this attraction to screentime may exist. First, it may be that the barriers to physical activity steer children with ASD to screens; if participation with our most popular physical activities are too socially, physically, and sensory demanding, turning to our most popular sedentary activities - screen-based activities - is understandable. Second, parents in research by Nally et al., (2000) spoke of television and video games as a way of managing behaviors. Finally, due to the visually-orientated nature of ASD, the attraction to video games and television may be greater (Must et al., 2015). Future research should be cognizant of the child's preference for video games when considering the development of exercise interventions for youth with ASD. Previous research has successfully incorporated "exer-gaming" as a means of significantly reducing repetitive behaviors in youth with ASD (Anderson-Hanley, Tureck, \& Schneiderman, 2011). Research should continue to examine their use as a means to increase physical activity levels of this population. That being said, echoing the call made from previous similar research (e.g. Stanish et al., 2017), researchers and practitioners should continue to strive for inclusive programming in school and community sport programming, as hobby preferences reported by the children with ASD were varied within the group, and many interests were similar to their TD peers.

\section{Limitations and future research}

There are some limitations within the current study that must be acknowledged and overcome in future research. First, this study employed a relatively small sample of children, especially when compiling frequency data on the hobby preferences of children with ASD. While the current study provides insight into these hobby preferences, more research is needed to provide a clearer view of the true desires of such a diverse population, including examining activity preference by severity of ASD, cognitive functioning, gender, and location (e.g. rural versus urban). Second, the

questionnaire utilized in this study took geographical and cultural activities or hobbies, such as hurling or camogie, into consideration. This limits the application of the results of this study to similar geographical regions and requires that future research examine the hobby preferences of children with ASD in other regions and cultures. Third, similar to previous research on this topic (e.g. Stanish et al., 2017), this study considered eliciting the perspective of the children with ASD an important aspect of this study, particularly relating to their hobby preference. However, reliance on self-reported data, particularly from children of such a young age, may be prone to bias. Future research should seek to garner this information from multiple sources, including parental reports.

\section{Conclusions}

The current study's primary finding is that children diagnosed with ASD report more sedentary hobby preferences than their TD peers. Over $60 \%$ of children with ASD preferred a sedentary hobby; this was in stark contrast to the 75\% of TD children who preferred an active hobby. Interestingly, physical activity participation, among the children with ASD, did not vary by hobby preference. The popularity of video game playing also emerged in the current study. The influence of hobby preferences, and the integration of child hobby preferences into physical activity interventions, is recommended for future research. 


\section{Perspectives}

The current study's main findings reflect that of Stanish et al., (2015), demonstrating sedentary hobbies to be preferred among children with ASD. A unique finding, that extends upon previous research, is that physical activity levels of the 9-years-olds with ASD participating in the current study did not vary by hobby preference (sedentary versus active). This finding requires further explorations to better understand the factors that are influencing physical activity participation among this population. Furthermore, the current study provides a list of hobby preferences reported by children with ASD; previously this information was limited to US children (e.g. Stanish et al., 2015).

\section{Author affiliations:}

1 Department of Kinesiology and Recreation Administration, Humboldt State University. Smr148@humboldt.edu

2 Department of Behavioral Health and Nutrition, University of Delaware. healys@udel.edu

${ }^{3}$ Department of Kinesiology and Recreation Administration, Humboldt State University. Reb22@humboldt.edu

* Correspondence: healys@udel.edu; Tel.:+1 302-831-3997

Author Contributions: Conceptualization: SH \& SR, Methodology and Formal Analysis: SH \& RB, Writing, review, and editing: SH, SR, \& RB.

Funding: This research study received no external funding

Conflicts of Interest: The authors declare no conflict of interest.

Acknowledgements: The authors would like to acknowledge the Irish Department of Children and Youth Affairs (DCYA) and Ireland's Central Statistics Office (CSO) for the collection of the data.

\section{References}

Anderson-Hanley, C., Tureck, K., \& Schneiderman, R. L. (2011). Autism and exergaming: effects on repetitive behaviors and cognition. Psychology research and behavior management, 4, 129.

American Psychiatric Association. Diagnostic and Statistical Manual of Mental Disorders. 5th ed. Washington: American Psychiatric Association; 2013.

Cadogan, S. L., Keane, E., \& Kearney, P. M. (2014). The effects of individual, family and environmental factors on physical activity levels in children: a cross-sectional study. BMC Pediatrics, 14(1), 107.

Christensen, D. L. (2016). Prevalence and characteristics of autism spectrum disorder among children aged 8 years-autism and developmental disabilities monitoring network, 11 sites, United States, 2012. MMWR. Surveillance Summaries, 65.

Cohen, D., Pichard, N., Tordjman, S., Baumann, C., Burglen, L., Excoffier, E., ... \& Héron, D. (2005). Specific genetic disorders and autism: clinical contribution towards their identification. Journal of Autism and Developmental Disorders, 35(1), 103-116.

Corvey, K., Menear, K. S., Preskitt, J., Goldfarb, S., \& Menachemi, N. (2016). Obesity, physical activity and sedentary behaviors in children with an autism spectrum disorder. Maternal and Child Health Journal, 20(2), 466-476.

Fournier, K. A., Hass, C. J., Naik, S. K., Lodha, N., \& Cauraugh, J. H. (2010). Motor coordination in autism spectrum disorders: a synthesis and meta-analysis. Journal of Autism and Developmental Disorders, 40(10), 1227-1240.

Godin, G., Jobin, J., \& Bouillon, J. (1986). Assessment of leisure time exercise behavior by self-report: A concurrent validity study. Canadian Journal of Public Health, 77, 359-361.

Godin, G., \& Shephard, R. J. (1985). A simple method to assess exercise behavior in the community. Canadian Journal of Applied Sport Science, 10(3), 141-146.

Healy, S., Haegele, J. A., Grenier, M., \& Garcia, J. M. (2017). Physical activity, screen-time behavior, and obesity among 13-year olds in ireland with and without autism spectrum disorder. Journal of autism and developmental disorders, 47(1), 49-57.

Healy, S., Msetfi, R., \& Gallagher, S. (2013). 'Happy and a bit Nervous': the experiences of children with autism in physical education. British Journal of Learning Disabilities, 41(3), 222-228.

Hill, G. M., \& Cleven, B. (2005). A comparison of students' choices of 9th grade physical education activities by ethnicity. The High School Journal, 89(2), 16-23.

Hill, G. M., \& Hannon, J. C. (2008). An analysis of middle school student's physical education physical activity preferences. Physical Educator, 65(4), 180. 
Macdonald, M., Esposito, P., \& Ulrich, D. (2011). The physical activity patterns of children with autism. BMC Research Notes, 4(1), 422.

Memari, A. H., Panahi, N., Ranjbar, E., Moshayedi, P., Shafiei, M., Kordi, R., \& Ziaee, V. (2015). Children with autism spectrum disorder and patterns of participation in daily physical and play activities. Neurology Research International, 531906, 1-7.

Memari, A. H., Ziaee, V., Shayestehfar, M., Ghanouni, P., Mansournia, M. A., \& Moshayedi, P. (2013). Cognitive flexibility impairments in children with autism spectrum disorders: links to age, gender and child outcomes. Research in Developmental Disabilities, 34(10), 3218-3225.

Murray, A., McCrory, C., Thornton, M., Williams, J., Quail, A., Swords, L., ... \& Harris, E. (2011). Growing Up in Ireland: Design, instrumentation and procedures for the child cohort. Ireland, Dublin: Department of Health and Children.

Must, A., Phillips, S. M., Curtin, C., Anderson, S. E., Maslin, M., Lividini, K., \& Bandini, L. G. (2014). Comparison of sedentary behaviors between children with autism spectrum disorders and typically developing children. Autism, 18(4), 376-384.

Must, A., Phillips, S., Curtin, C., \& Bandini, L. G. (2015). Barriers to physical activity in children with autism spectrum disorders: relationship to physical activity and screen time. Journal of Physical Activity and Health, 12(4), 529-534.

Nally, B., Houlton, B., \& Ralph, S. (2000). Researches in brief: The management of television and video by parents of children with autism. Autism, 4(3), 331-337.

Obrusnikova, I., \& Cavalier, A. R. (2011). Perceived barriers and facilitators of participation in after-school physical activity by children with autism spectrum disorders. Journal of Developmental and Physical Disabilities, 23(3), 195-211.

Obrusnikova, I., \& Miccinello, D. L. (2012). Parent perceptions of factors influencing after-school physical activity of children with autism spectrum disorders. Adapted Physical Activity Quarterly, 29(1), 63-80.

Pan, C. Y., \& Frey, G. C. (2006). Physical activity patterns in youth with autism spectrum disorders. Journal of Autism and Developmental Disorders, 36(5), 597-606.

Pan, C.-Y., Tsai, C.-L., Chu, C.-H., \& Hsieh, K.-W. (2011). Physical activity and self-determined motivation of adolescents with and without autism spectrum disorders in inclusive physical education. Research in Autism Spectrum Disorders, 5, 733-741.

Sallis, J. F., Buono, M. J., Roby, J. J., Micale, F. G., \& Nelson, J. A. (1993). Seven-day recall and other physical activity self-reports in children and adolescents. Medicine and Science in Sports and Exercise, 25(1), 99-108.

Stanish, H., Curtin, C., Must, A., Phillips, S., Maslin, M., \& Bandini, L. (2015). Enjoyment, barriers, and beliefs about physical activity in adolescents with and without autism spectrum disorder. Adapted Physical Activity Quarterly, 32(4), 302-317.

Stanish, H. I., Curtin, C., Must, A., Phillips, S., Maslin, M., \& Bandini, L. G. (2017). Physical activity levels, frequency, and type among adolescents with and without autism spectrum disorder. Journal of autism and developmental disorders, 47(3), 785-794.

Tyler, K., MacDonald, M., \& Menear, K. (2014). Physical activity and physical fitness of school-aged children and youth with autism spectrum disorders. Autism Research and Treatment, 2014.

Welk, G. J., Wood, K., \& Morss, G. (2003). Parental influences on physical activity in children: An exploration of potential mechanisms. Pediatric Exercise Science, 15(1), 19-33.

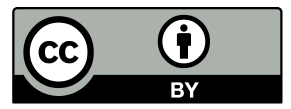

(C) 2018 by the authors. Submitted for possible open access publication under the terms and conditions of the Creative Commons Attribution (CC BY) license (http://creativecommons.org/licenses/by/4.0/). 